Bull. Austral. Math. Soc.

VOL. 41 (1990) [347-354]

\title{
ON PERIODIC SOLUTIONS TO AUTONOMOUS RETARDED FUNCTIONAL DIFFERENTIAL EQUATIONS
}

\author{
ZHANYUAN HOU
}

\begin{abstract}
Under the assumption that $C_{a}=C\left([-r, 0], S^{n-1}(a)\right)$ is positively invariant for $a>0$, two necessary and sufficient conditions are obtained for an autonomous retarded functional differential equation to have a non-trivial periodic solution in $C_{a}$. Moreover, a feasible sufficient condition is given, which is better for $n=2$ than that given by Dos Reis and Baroni.
\end{abstract}

\section{INTRODUCTION}

Let $\boldsymbol{R}$ be the set of real numbers, $\mathbf{R}^{+}$the set of non-negative numbers and $\mathbf{F}^{n}$ the real Euclidean space. For $r \geqslant 0$ let $C=C\left([-r, 0], \mathrm{R}^{n}\right)$ be the space of continuous functions from $[-r, 0]$ to $R^{n}$ with the topology of uniform convergence given by the norm $\|\phi\|=\sup _{-r \leqslant \theta \leqslant 0}|\phi(\theta)|$.

Consider the autonomous retarded functional differential equation

$$
X^{\prime}(t)=f\left(X_{t}\right)
$$

where $f: C \rightarrow \mathbf{R}^{n}$ is a continuous map that takes bounded sets into bounded sets and $X_{t}$ is defined as $X_{t}(\theta)=X(t+\theta)$ for $-r \leqslant \theta \leqslant 0$. Suppose that unicity and continuity with respect to initial values hold and that the solutions are defined on $[-r, \infty)$. Then equation (1) defines a semi-dynamical system $\pi: C \times \mathrm{R}^{+} \rightarrow C$ given by $\pi(\phi, t)=X_{t}(\phi)$.

Let $X\left(t, t_{0}, \phi\right)$ be a solution to $(1)$ on $[-r, A)$ with $X_{t_{0}}\left(t_{0}, \phi\right)=\phi$. For the sake of convenience, we denote the solution $X(t, 0, \phi)$ by $X(t, \phi)$ and $X_{t}(0, \phi)$ by $X_{t}(\phi)$. A solution $X(t, \phi)$ defined on $[-r, \infty)$ is called periodic if there is a $T>0$ such that $\phi=\pi(\phi, T)$, or equivalent, $\pi(\phi, t)=\pi(\phi, t+T)$ for all $t \geqslant 0$. A set $M \subseteq C$ is called positively invariant if $\pi(\phi, t) \in M$ holds for all $\phi \in M$ and $t \geqslant 0$.

For $a>0$, let $S^{n-1}(a)=\left\{x \in \mathbf{R}^{n}:|x|=a\right\}$ and $C_{a}=C\left([-r, 0], S^{n-1}(a)\right)$. Suppose that $C_{a}$ is positively invariant. The problems which concern us are these: Does (1) necessarily have periodic solutions in $C_{a}$ ? What is the essential condition

Received 23 May 1989

The author thanks J. Cassel for useful discussions and suggestions and U. Ehrenmark for much concern during the visit.

Copyright Clearance Centre, Inc. Serial-fee code: 0004-9729/90 \$A2.00+0.00. 
ensuring that (1) has a non-trivial periodic solution in $C_{a}$ ? Many examples (see [3], p.326) have shown that there may not be any periodic solutions to (1) although $C_{a}$ is positively invariant. Thus the first problem has already been solved. In the case of $n=2,[1]$ presented not only a good result but also a better method for the solution of the second problem. The aim of this paper is to generalise the result in [1] to all cases of $n \geqslant 2$. Indeed, we shall give necessary and sufficient conditions for (1) to have a non-trivial periodic solution in $C_{a}$.

\section{MAIN RESULTS}

THEOREM 1. Suppose that $f: C \rightarrow \mathbf{R}^{n}$ is a continuous map that takes bounded sets into bounded sets. Let $\pi$ be the semi-dynamical system defined by (1). If $C_{a}$ is positively invariant, then (1) has a non-trivial periodic solution in $C_{a}$ if and only if there is a closed continuous curve $L_{a}$ on $S^{n-1}(a)$, a positively invariant closed set $C_{L} \subseteq C\left([-r, 0], L_{a}\right) \subseteq C_{a}$, a point $X_{0} \in L_{a}$ and a $T_{1}>0$ satisfying:

(i) $J=\left\{\phi \in C_{L}: \phi(0)=X_{0}\right\}$ is non-empty;

(ii) $f(\phi) \neq 0$ holds for all $\phi \in J$;

(iii) for any $\phi \in J$, there is a $t^{\prime} \in\left(0, T_{1}\right]$ such that $X\left(t^{\prime}, \phi\right)=X_{0}$.

To prove Theorem 1 , the following lemmas are needed.

Lemma 1. Let $r$ be the delay in (1). If the operator $\pi^{t}: C \rightarrow C$ defined by $\pi^{t}(\phi)=\pi(\phi, t)$ takes bounded sets into bounded sets, then for any bounded set $B \subset C$ and $t \geqslant r, \operatorname{cl}\left(\pi^{t} B\right)$ (closure of $\pi^{t} B$ ) is compact. Moreover, $\operatorname{cl}\left(\bigcup_{r \leqslant t \leqslant \bar{t}} \pi^{t} B\right)$ is also compact for any $\bar{t} \geqslant r$.

Proof: By the Ascoli-Arzela theorem, the compactness of $\mathrm{cl}\left(\pi^{t} B\right)$ can easily be proved for $t \geqslant r$. This, together with continuity, implies that for any $t_{0} \in[r, \bar{t}]$, $\lim _{t \rightarrow t_{0}} \pi^{t} \phi=\pi^{t_{0}} \phi$ holds uniformly for $\phi \in B$. Suppose $\left\{\psi_{n}\right\} \subseteq \bigcup_{r \leqslant t \leqslant \bar{t}} \pi^{t} B$. We will show that there is a convergent subsequence $\left\{\psi_{n_{k}}\right\} \subseteq\left\{\psi_{n}\right\}$. Clearly there are $\left\{\phi_{n}\right\} \subseteq B$ and $\left\{t_{n}\right\} \subseteq[r, \bar{t}]$ such that $\pi^{t_{n}} \phi_{n}=\psi_{n}$ for $n=1,2, \ldots$. By the compactness of $[r, \bar{t}]$, we may suppose without loss of generality that $\lim _{n \rightarrow \infty} t_{n}=t_{0}$. Then $\pi^{t_{0}} \phi_{n} \varepsilon \pi^{t_{0}} B$ corresponds to $\pi^{t_{n}} \phi_{n} \varepsilon \pi^{t_{n}} B$. Because $\lim _{t \rightarrow t_{0}} \pi^{t} \phi_{n}=\pi^{t_{0}} \phi_{n}$ holds uniformly for $n$, for any $\varepsilon>0$, there is a natural number $N$ such that

$$
\left\|\pi^{t_{n}} \phi_{n}-\pi^{t_{0}} \phi_{n}\right\|<\frac{\varepsilon}{2}
$$

holds for any $n>N$. Then the compactness of $\operatorname{cl}\left(\pi^{t_{0}} B\right)$ implies that there exists a subsequence $\left\{\pi^{t_{0}} \phi_{n_{k}}\right\} \subseteq\left\{\pi^{t_{0}} \phi_{n}\right\}$ and a $\psi_{0} \in \operatorname{cl}\left(\pi^{t_{0}} B\right)$ such that $\lim _{k \rightarrow \infty} \pi^{t_{0}} \phi_{n_{k}}=\psi_{0}$. 
We assume that $\left\|\pi^{t_{0}} \phi_{n_{k}}-\psi_{0}\right\|<\varepsilon / 2$ for $k>k_{1}$, where $n_{k_{1}}>N$ holds. Then,

$$
\left\|\psi_{n_{k}}-\psi_{0}\right\| \leqslant\left\|\pi^{t_{n_{k}}} \phi_{n_{k}}-\pi^{t_{0}} \phi_{n_{k}}\right\|+\left\|\pi^{t_{0}} \phi_{n_{k}}-\psi_{0}\right\|<\varepsilon
$$

holds for $k>k_{1}$. Thus, $\lim _{k \rightarrow \infty} \psi_{n_{k}}=\psi_{0}$. Namely, $\mathrm{cl}\left(\bigcup_{r \leqslant t \leqslant \bar{t}} \pi^{t} B\right)$ is compact.

Lemma 2. Suppose $E$ is a Banach space, $K$ either a cone or a truncated cone in $E, G \subseteq E$ an open bounded set with $0 \in G$ and $\partial G$ the boundary of $G$. If $A: \partial G \cap K \rightarrow K$ is completely continuous and $\inf \{\|A \phi\|: \phi \in \partial G \cap K\}>0$, then $A$ has an eigenvector in $\partial G \cap K$.

Lemma 3. Let $F \subseteq E$ be a bounded, closed and convex set with $0 \notin F$. Then the set $K(F)=\{x \in E:(\exists z \in F)(\exists t \geqslant 0)(x=t z)\}$ is a cone in $E$.

The proofs of Lemma 2 and Lemma 3 can be found in $[1,2,4]$.

Proof of Theorem 1:

NECESSITY. Let $X\left(t, \phi_{0}\right)$ be a non-trivial periodic solution in $C_{a}$. Then there is a $T>0$ such that $X\left(t+T, \phi_{0}\right)=X\left(t, \phi_{0}\right)$ holds for all $t \geqslant 0$. Let $L_{a}=\left\{X\left(t, \phi_{0}\right): 0 \leqslant\right.$ $t \leqslant T\}$; then $L_{a}$ is a closed continuous curve on $S^{n-1}(a)$. It is obvious that $C_{L}=$ $\left\{X_{t}\left(\phi_{0}\right): 0 \leqslant t \leqslant T\right\} \subseteq C\left([-r, 0], L_{a}\right)$ is positively invariant. Because $X\left(t, \phi_{0}\right)$ is nontrivial, there is a $t_{0} \in[0, T]$ such that $d /\left.d t X\left(t, \phi_{0}\right)\right|_{t_{0}} \neq 0$, that is $f\left(X_{t_{0}}\left(\phi_{0}\right)\right) \neq 0$. We denote $X\left(t_{0}, \phi_{0}\right)$ by $X_{0}$; then we have $J=\left\{\phi \in C_{L}: \phi(0)=X_{0}\right\}=\left\{X_{t_{0}}\left(\phi_{0}\right)\right\}$. Thus $f(\phi) \neq 0$ holds for $\phi \in J$. Let $T_{1}=T$ and $t^{\prime}=T_{1}$. Then $X\left(t^{\prime}, \phi\right)=X_{0}$ for any $\phi \in J$. Hence the necessity holds.

Sufficiency. For $r=0$ the result is trivial, so we assume $r>0$.

Let $F=\left\{\phi \in C: \phi(0)=X_{0}\right.$ and $\left.\|\phi\| \leqslant 2 a\right\}$. It is clear that $F$ is closed, bounded, convex and $0 \notin F$. Then Lemma 3 implies that $K(F)$ is a cone and $K=K(F) \cap\{\phi \in$ $C:\|\phi\| \leqslant a\}$ a truncated cone in $C$. Let

$$
G=\{\phi \in C:\|\phi\|<2 a\}-\left\{\phi \in C:\left(\exists \phi_{1} \in C_{L}\right)(\exists \alpha \in[1,3])\left(\phi=\alpha \phi_{1}\right)\right\} .
$$

Then $G$ is an open bounded set and $K \cap \partial G=\left\{\phi \in C_{L}: \phi(0)=X_{0}\right\}=J$.

We define the map $\tau: J \rightarrow[r, \infty)$ by $\tau(\phi)=\inf \{t \geqslant r: \pi(\phi, t) \in J\}$. Then (iii) implies $r \leqslant \tau(\phi) \leqslant\left(k_{0}+1\right) T_{1}$ for all $\phi \in J$ and some natural number $k_{0}$ with $k_{0} T_{1} \geqslant r$. We assert that $\tau$ is continuous in $J$. In fact, for any $\phi_{n}, \bar{\phi} \in J$ and $t_{n}=\tau\left(\phi_{n}\right), \bar{t}=\tau(\phi)$ with $\phi_{n} \rightarrow \bar{\phi}$ as $n \rightarrow \infty$, we only need to show $t_{n} \rightarrow \bar{t}$ as $n \rightarrow \infty$. By (ii) we know that $d /\left.d t X(t, \bar{\phi})\right|_{\bar{t}}=f\left(X_{\bar{t}}(\phi)\right) \neq 0$, which, together with the definition of $\tau$, implies the existence of $s_{1} \in[0, r)$ and $s_{2}>\bar{t}$ such that $X(t, \bar{\phi}) \neq X_{0}$ holds on $\left[s_{1}, s_{2}\right]$ except at $t=\bar{t}$. By continuity, $X\left(t, \phi_{n}\right) \rightarrow X(t, \bar{\phi})$ 
holds uniformly on $\left[s_{1}, s_{2}\right]$ as $n \rightarrow \infty$. Because $\left\{X(t, \bar{\phi}): s_{1} \leqslant t \leqslant s_{2}\right\}$, as well as $\left\{X\left(t, \phi_{n}\right): s_{1} \leqslant t \leqslant s_{2}\right\}$, is a section of the curve $L_{a}$, we must have $t_{n} \in\left[s_{1}, s_{2}\right]$ when $n$ is large enough. If $t_{n} \nrightarrow \bar{t}$ as $n \rightarrow \infty$, then there is a subsequence $\left\{t_{n_{k}}\right\}$ such that $t_{n_{k}} \rightarrow \overline{\bar{t}} \in\left[s_{1}, s_{2}\right]$ as $k \rightarrow \infty$ but $\overline{\bar{t}} \neq \bar{t}$. Thus $X_{0}=X\left(t_{n_{k}}, \phi_{n_{k}}\right) \rightarrow X(\overline{\bar{t}}, \bar{\phi}) \neq X_{0}$ as $k \rightarrow \infty$, which is a contradiction. Therefore we have $t_{n} \rightarrow \bar{t}$ as $n \rightarrow \infty$.

Let $A: J \rightarrow C$ be defined by $A \phi=\pi(\phi, \tau(\phi))$. Then $A J \subseteq J$. Since $\pi(\phi, t)$ is continuous in $(\phi, t)$ and $\tau(\phi)$ is continuous in $\phi, A$ is continuous. Clearly, we have $A J \subseteq \bigcup_{\tau \leqslant t \leqslant\left(t_{0}+1\right) T_{1}} \pi^{t} J$ which, by Lemma 1 , implies that $A$ is completely continuous. Moreover, $\inf \{\|A \phi\|: \phi \in J\}=a>0$ holds. By Lemma 2, there exist a $\phi_{0} \in J$ and a $\mu \in R$ such that $A \phi_{0}=\mu \phi_{0}$. As $A \phi_{0} \in J$, we have $\left\|\phi_{0}\right\|=a=\left\|A \phi_{0}\right\|=\left\|\mu \phi_{0}\right\|=$ $|\mu| \cdot\left\|\phi_{0}\right\|$ thus, $|\mu|=1$.

By the definition of a cone $[1], \phi_{0}$ and $-\phi_{0}$ cannot both belong to $K$. Thus $\mu=1$, that is $A \phi_{0}=\phi_{0}$. Since $f(\phi) \neq 0$ holds on $J$, it is obvious that $X_{t}\left(\phi_{0}\right)$ is a non-trivial periodic solution.

THEOREM 2. Under the same general assumption as above, (1) has a non-trivial periodic solution in $C_{a}$ if and only if there is a closed continuous curve $L_{a}$ on $S^{n-1}(a)$, a positively invariant compact set $C_{L} \subseteq C\left([-r, 0], L_{a}\right) \subseteq C_{a}$ and a point $X_{0} \in L_{a}$ satisfying:

(i) $J=\left\{\phi \in C_{L}: \phi(0)=X_{0}\right\}$ is not empty;

(ii) $f(\phi) \neq 0$ holds for any $\phi \in J$;

(iii) for any $\phi \in J$, there is a $t^{\prime}>0$ such that $X_{t^{\prime}}(\phi) \in J$.

Proof: From (i) - (iii) we know that for any $\phi \in J$, there is a $t^{\prime}(\phi)>0$ such that $X_{t^{\prime}}(\phi) \in J$ but that $X_{t}(\phi) \notin J$ for $0<t<t^{\prime}$. Suppose $\left\{t^{\prime}(\phi): \phi \in J\right\}$ is unbounded. Then by the compactness of $C_{L}$, we can select a sequence $\left\{\phi_{n}\right\} \subseteq J$ and a corresponding $\left\{t_{n}\right\}=\left\{t_{n}\left(\phi_{n}\right)\right\}$ such that both $\phi_{n} \rightarrow \phi_{0} \in J$ and $t_{n} \rightarrow \infty$ hold as $n \rightarrow \infty$. By the continuity with respect to initial values, we conclude that $X_{t}\left(\phi_{0}\right) \notin J$ for all $t \geqslant 0$, which contradicts (iii). Hence $\left\{t^{\prime}(\phi): \phi \in J\right\}$ is a bounded set. By Theorem 1, (1) has a non-trivial periodic solution. Thus the sufficiency is proved. The necessity is obvious from Theorem 1.

THEOREM 3. Under the same general assumption as above, if there is a closed continuous curve $L_{a}$ on $S^{n-1}(a)$ such that $C\left([-r, 0], L_{a}\right) \subseteq C_{a}$ is positively invariant and $f(\phi) \neq 0$ holds for $\phi \in C\left([-r, 0], L_{a}\right)-B$, then (1) has a non-trivial periodic solution in $C_{a}$. Here

$$
B=\left\{\phi \in C\left([-r, 0], L_{a}\right): f(\phi)=0, \phi(s) \text { is not constant for } s \in[-r, 0]\right\}
$$
belongs to one of the following cases:

(i) $B$ is empty; 
(ii) $B$ is a finite set;

(iii) all $\phi \in B$, except for a finite number of elements of $B$, satisfy $\phi(s) \rightarrow$ $\phi(0)(s \rightarrow 0)$ along $L_{a}$ in the same direction. Moreover, $\{\phi(0): \phi \in B\} \neq$ $L_{\mathbf{a}}$.

Proof: Suppose that $B$ satisfies (iii). If there is a $\phi_{0} \in B$ and a $\phi \in$ $C\left([-\tau, 0], L_{a}\right)$ such that $X_{t^{\prime}}(\phi)=\phi_{0}=X_{t^{\prime \prime}}(\phi)$ holds for some $t^{\prime \prime}>t^{\prime} \geqslant 0$, then $X_{t}(\phi)$ is a non-trivial periodic solution as $\phi_{0}(s)$ is not constant. If no such solution exists, we first show that for any $X_{0} \in L_{a}$ and any $\phi \in C\left([-r, 0], L_{a}\right)$, there is a $t^{\prime}>0$ such that $X\left(t^{\prime}, \phi\right)=X_{0}$.

In fact, $X(t, \phi)$ obviously exists on $[0, \infty)$ and moves along $L_{a}$. For convenience, we denote one direction of $L_{a}$ by $(+)$ and the other by $(-)$. Suppose that all $\phi \in B$, except for a finite number of elements of $B$, are in $(+)$. If $X(t, \phi)$ changes direction at some $\bar{t}>0$, then $X^{\prime}(\bar{t}, \phi)=0$ holds, that is $f\left(X_{\bar{t}}(\phi)\right)=0$, which implies $X_{\bar{t}}(\phi) \in B$ since $f(\phi) \neq 0$ on $C\left([-r, 0], L_{a}\right)-B$. If $X(t, \phi)$ changes direction infinitely often, then there is a sequence $\left\{t_{n}\right\}$ such that $t_{n+1}>t_{n}>0, X_{t_{n}}(\phi) \in B, X_{t_{2 n+1}}(\phi)$ in (+) and $X_{t_{2 n}}(\phi)$ in $(-)$ hold for all positive integers $n$. Since $X_{t}(\phi)$ cannot coincide with any element of $B$ more than once, we have $X_{t_{i}}(\phi) \neq X_{t_{j}}(\phi)$ for $i \neq j$. Therefore $B$ has an infinite number of elements that are in $(-)$. This contradicts the assumption. Thus $X(t, \phi)$ changes direction at most a finite number of times. Hence there must be a $T_{1}>0$ such that $X(t, \phi)$ moves along $L_{a}$ in a definite direction for $t \geqslant T_{1}$. If $X(t, \phi) \rightarrow X_{1} \in L_{a}$ as $t \rightarrow \infty$, then $f\left(X_{t}(\phi)\right) \rightarrow 0$ as $t \rightarrow \infty$. Because $f$ is continuous and $X_{t}(\phi) \rightarrow \phi_{1}$ as $t \rightarrow \infty$, where $\phi_{1}(s)=X_{1}$ for $s \in[-r, 0]$, we have $f\left(\phi_{1}\right)=0$ which implies $\phi_{1} \in B$. This contradicts the assumption too. Thus $\lim _{t \rightarrow \infty} X(t, \phi)$ does not exist. Therefore there must be a $t^{\prime}>T_{1}$, such that $X\left(t^{\prime}, \phi\right)=X_{0}$.

Suppose $\phi_{0} \in C\left([-r, 0], L_{a}\right)$ and $\nu\left(\phi_{0}\right)=\left\{X_{t}\left(\phi_{0}\right): t \geqslant 0\right\}$. Let $\omega\left(\phi_{0}\right)$ be the limit set of $\nu\left(\phi_{0}\right)$. Then $\omega\left(\phi_{0}\right) \subseteq C\left([-r, 0], L_{a}\right)$ is non-empty, compact and positively invariant [3]. By the above conclusion we know that $J=\left\{\phi \in \omega\left(\phi_{0}\right): \phi(0)=X_{0}\right\}$ is non-empty for any $X_{0} \in L_{a}$. By $\{\phi(0): \phi \in B\} \neq L_{a}$ we can choose $X_{0} \in L_{a}-\{\phi(0)$ : $\phi \in B\}$ so that $J \cap B$ is empty. Thus, $f(\phi) \neq 0$ holds on $J$. Furthermore, for any $\phi \in J$, there is a $t^{\prime}>0$ such that $X_{t^{\prime}}(\phi) \in J$. By Theorem 2, (1) has a non-trivial periodic solution in $C_{a}$.

If $B$ satisfies either (i) or (ii), the proof is similar to the above.

\section{REMARKS AND EXAMPLES}

REMARK. Our results generalise the theorem in [1] in the following aspects. Firstly, [1] dealt only with the case of $n=2$, while our results can be used for all cases of $n \geqslant 2$. Secondly, [1] presented only a sufficient condition, whereas our results include necessary and sufficient conditions, which are normally regarded as the best solution to 
any problem. Even our sufficient condition (Theorem 3) is much better than that of [1] because the theorem in [1] is only the special case of Theorem 3, when $n=2$ and $B$ satisfies (i). Thus, our results can be used for a more general class of equations than [1] can.

EXample 1. Consider the system

$$
\left\{\begin{array}{l}
X_{1}^{\prime}(t)=X_{3}(t) \int_{-1}^{0}\left[X_{1}(t+\theta)-\sin \theta\right]^{2} d \theta\left[1+X_{2}^{2}\left(t-\frac{1}{2}\right)+X_{3}^{2}(t-1)\right] \\
X_{2}^{\prime}(t)=2 X_{1}(t) X_{3}(t) \int_{-1}^{0}\left[X_{1}(t+\theta)-\sin \theta\right]^{2} d \theta\left[1+X_{2}^{2}\left(t-\frac{1}{2}\right)+X_{3}^{2}(t-1)\right] \\
X_{3}^{\prime}(t)=-X_{1}(t)\left[1+2 X_{2}(t)\right] \int_{-1}^{0}\left[X_{1}(t+\theta)-\sin \theta\right]^{2} d \theta\left[1+X_{2}^{2}\left(t-\frac{1}{2}\right)+X_{3}^{2}(t-1)\right]
\end{array}\right.
$$

Let $X=\left(X_{1}, X_{2}, X_{3}\right)^{T}, f=\left(f_{1}, f_{2}, f_{3}\right)^{T}$,

$$
\begin{aligned}
& f_{1}(\phi)=\phi_{3}(0) \int_{-1}^{0}\left[\phi_{1}(\theta)-\sin \theta\right]^{2} d \theta\left[1+\phi_{2}^{2}\left(-\frac{1}{2}\right)+\phi_{3}^{2}(-1)\right] \\
& f_{2}(\phi)=2 \phi_{1}(0) \phi_{3}(0) \int_{-1}^{0}\left[\phi_{1}(\theta)-\sin \theta\right]^{2} d \theta\left[1+\phi_{2}^{2}\left(-\frac{1}{2}\right)+\phi_{3}^{2}(-1)\right] \text { and } \\
& f_{3}(\phi)=-\phi_{1}(0)\left[1+2 \phi_{2}(0)\right] \int_{-1}^{0}\left[\phi_{1}(\theta)-\sin \theta\right]^{2} d \theta\left[1+\phi_{2}^{2}\left(-\frac{1}{2}\right)+\phi_{3}^{2}(-1)\right] .
\end{aligned}
$$

Then (3.1) can be written as $X^{\prime}(t)=f\left(X_{t}\right)$. It is easy to verify that $d / d t\left[X_{1}^{2}(t)+\right.$ $\left.X_{2}^{2}(t)+X_{3}^{2}(t)\right]=0, d / d t\left[X_{2}(t)-X_{1}^{2}(t)\right]=0$ and the general assumptions hold for (3.1). Let $L_{a} \subseteq S^{2}(a)$ be defined by $X_{1}^{2}+X_{2}^{2}+X_{3}^{2}=a^{2}$ with $X_{2}=X_{1}^{2}$. Then $C_{a}$, as well as $C\left([-1,0], L_{a}\right) \subset C_{a}$, is positively invariant. If $a \geqslant \sqrt{\left(\sin ^{2} 1+1 / 2\right)^{2}-1 / 4}$ holds, we denote

$$
\begin{aligned}
& \phi_{0}(s)=\left(\sin s, \sin ^{2} s, \sqrt{a^{2}-\sin ^{2} s-\sin ^{4} s}\right) \\
& \bar{\phi}_{0}(s)=\left(\sin s, \sin ^{2} s,-\sqrt{a^{2}-\sin ^{2} s-\sin ^{4} s}\right)
\end{aligned}
$$

and $B=\left\{\phi_{0}, \bar{\phi}_{0}\right\}$. Then $f(\phi) \neq 0$ holds for $\phi \in C\left([-1,0], L_{a}\right)-B$. If $0<a<\sqrt{\left(\sin ^{2} 1+1 / 2\right)^{2}-1 / 4}$, then $f(\phi) \neq 0$ holds for all $\phi \in C\left([-1,0], L_{a}\right)$. By Theorem 3, (3.1) has a non-trivial periodic solution in $C_{a}$ for any $a>0$. 
Example 2. Consider the system

$$
\left\{\begin{aligned}
X_{1}^{\prime}(t)= & X_{3}(t) \int_{-1}^{0}\left\{\left[X_{1}(t+\theta)-\sin \theta\right]^{2}\right. \\
& \left.+\left[X_{3}(t+\theta)-\sqrt{4-\sin ^{2} \theta-\sin ^{4} \theta}\right]^{2}\right\} d \theta\left[1+X_{3}^{2}(t-2)\right] \\
X_{2}^{\prime}(t)= & 2 X_{1}(t) X_{3}(t) \int_{-1}^{0}\left\{\left[X_{1}(t+\theta)-\sin \theta\right]^{2}\right. \\
& \left.+\left[X_{3}(t+\theta)-\sqrt{4-\sin ^{2} \theta-\sin ^{4} \theta}\right]^{2}\right\} d \theta\left[1+X_{3}^{2}(t-2)\right] \\
X_{3}^{\prime}(t)= & X_{1}(t)\left[1+2 X_{2}(t)\right] \int_{-1}^{0}\left\{\left[X_{1}(t+\theta)-\sin \theta\right]^{2}\right. \\
& \left.+\left[X_{3}(t+\theta)-\sqrt{4-\sin ^{2} \theta-\sin ^{4} \theta}\right]^{2}\right\} d \theta\left[1+X_{3}^{2}(t-2)\right]
\end{aligned}\right.
$$

Let $L_{a} \subseteq S^{2}(a)$ be defined by $X_{1}^{2}+X_{2}^{2}+X_{3}^{2}=a^{2}$ with $X_{2}=X_{1}^{2}$. Then both $C_{a}$ and $C\left([-2,0], L_{a}\right)$ are positively invariant. If $a \neq 2(a>0)$, then $f(\phi) \neq 0$ holds for all $\phi \in C\left([-2,0], L_{a}\right)$. If $a=2$, we put

$$
B=\left\{\phi \in C\left([-2,0], L_{2}\right): \phi(\theta)=\left(\sin \theta, \sin ^{2} \theta, \sqrt{4-\sin ^{2} \theta-\sin ^{4} \theta}\right) \text { for } \theta \in[-1,0]\right\} .
$$

Then $f(\phi) \neq 0$ holds for $\phi \in C\left([-2,0], L_{2}\right)-B$. Furthermore, for all $\phi \in B \phi(s) \rightarrow$ $\phi(0)(s \rightarrow 0)$ are in the same direction along $L_{2}$. By Theorem 3, (3.2) has a non-trivial periodic solution in $C_{a}$ for any $a>0$.

REMARK. Although the two examples above can be reduced to the case of $n=2$, they cannot be treated by the theorem of [1] because $f(\phi)$ may have zeros in $C_{a}$. Because Theorem 3 is only a sufficient condition, there exist systems that cannot be treated by this. In this case Theorems 1 and 2 may be helpful. The next example will show this.

EXAmple 3. Consider the system

$$
\left\{\begin{array}{l}
X_{1}^{\prime}(t)=X_{2}(t)\left(2 \pi \max _{-1 \leqslant s \leqslant 0}\left|X_{1}(t+s)\right|\right) /\left[\left|X_{1}(t-1)\right|+\left|X_{2}(t-1)\right|\right] \\
X_{2}^{\prime}(t)=-X_{1}(t)\left(2 \pi \max _{-1 \leqslant s \leqslant 0}\left|X_{1}(t+s)\right|\right) /\left[\left|X_{1}(t-1)\right|+\left|X_{2}(t-1)\right|\right]
\end{array}\right.
$$

for which $f=\left(f_{1}, f_{2}\right)^{T}, f_{1}=\phi_{2}(0)\left(2 \pi \max _{-1 \leqslant s \leqslant 0}\left|\phi_{1}(s)\right|\right) /\left(\left|\phi_{1}(-1)\right|+\left|\phi_{2}(-1)\right|\right), f_{2}=$ $-\phi_{1}(0)\left(2 \pi \max _{-1 \leqslant s \leqslant 0}\left|\phi_{1}(s)\right|\right) /\left(\left|\phi_{1}(-1)\right|+\left|\phi_{2}(-1)\right|\right)$ and $\phi=\left(\phi_{1}, \phi_{2}\right)^{T} \in C\left([-1,0], R^{2}\right)$. Clearly, the general assumptions hold for (3.3). Moreover, $C_{a}$ is positively invariant for any $a>0$ as $d / d t\left[X_{1}^{2}(t)+X_{2}^{2}(t)\right]=0$. Let $L_{a}=S^{1}(a)=\left\{X \in R^{2}: X_{1}^{2}+X_{2}^{2}=a^{2}\right\}$, 
then $C\left([-1,0], L_{a}\right)=C_{a}$. Let $\phi_{0}(s)=(a, 0)^{T}$ for $-1 \leqslant s \leqslant 0, X_{0}=(a, 0)^{T} \in L_{a}$, $\nu^{+}\left(\phi_{0}\right)=\left\{X_{t}\left(\phi_{0}\right): t \geqslant 0\right\}, C_{L}=\operatorname{cl}\left(\nu^{+}\left(\phi_{0}\right)\right)$ and $J=\left\{\phi \in C_{L}: \phi(0)=X_{0}\right\}$. It is easy to verify that $C_{L}=\nu^{+}\left(\phi_{0}\right) \cup \omega\left(\phi_{0}\right)^{[3]}\left[\omega\left(\phi_{0}\right)\right.$ is the positive limit set of $\nu^{+}\left(\phi_{0}\right)$ with $\pi^{t} \omega\left(\phi_{0}\right)=\omega\left(\phi_{0}\right)$ for any $\left.t \geqslant 0\right]$. By Lemma $1, C_{L}$ is a positively invariant compact set. It is obvious that $f(\phi) \neq 0$ holds on $J$. Furthermore, for any $\phi \in J$ and $t \geqslant 0$, the solution $X(t, \phi)$ satisfies

$$
\left\{\begin{array}{l}
X_{1}(t)=a \cos 2 \pi \int_{0}^{t}\left\{\max _{-1 \leqslant s \leqslant 0}\left|X_{1}(\ell+s)\right| /\left[\left|X_{1}(\ell-1)\right|+\left|X_{2}(\ell-1)\right| \mid\right\} d \ell\right. \\
\left.X_{2}(t)=-a \sin 2 \pi \int_{0}^{t}\left\{\max _{-1 \leqslant s \leqslant 0}\left|X_{1}(\ell+s)\right| /|| X_{1}(\ell-1)|+| X_{2}(\ell-1) \mid\right]\right\} d \ell .
\end{array}\right.
$$

Thus $\max _{-1 \leqslant \ell \leqslant 0}\left|X_{1}(\ell+s)\right|=a$ holds for $0 \leqslant \ell \leqslant 1$. Since

$$
a=|X(\ell-1)| \leqslant\left|X_{1}(\ell-1)\right|+\left|X_{2}(\ell-1)\right| \leqslant \sqrt{2}|X(\ell-1)|=\sqrt{2} a
$$

holds for any $\ell \geqslant 0$, we have

$$
\sqrt{2} \pi t \leqslant 2 \pi \int_{0}^{t}\left\{\max _{-1 \leqslant s \leqslant 0}\left|X_{1}(\ell+s)\right| /|| X_{1}(\ell-1)|+| X_{2}(\ell-1)||\right\} d \ell \leqslant 2 \pi t
$$

for $t \in[0,1]$. Therefore there exists a $t_{0} \in[1 / 2,1 / \sqrt{2}]$ such that $X_{1}\left(t_{0}\right)=-a$. Similarly, there is a $t^{\prime} \in\left[t_{0}+1 / 2, t_{0}+1 / \sqrt{2}\right]$ such that $X_{t^{\prime}}(\phi) \in J$. By Theorem 2 (3.3) has a non-trivial periodic solution in $C_{a}$.

\section{REFERENCES}

[1] J.G. Dos Reis and R.L.S. Baroni, 'On the existence of periodic solutions for autonomous retarded functional differential equations on $R^{2}$, Proc. Royal Soc. Edinburgh Section $A$ 102 (1986), 259-262.

[2] J.K. Hale, Functional Differential Equations, (Appl. Math. Sci. 3) (Springer-Verlag, Berlin, Heidelberg, New York, 1971).

[3] J.K. Hale, Theory of Functional Differential Equations, (2nd edition), (Appl. Math. Sci. 3) (Springer-Verlag, Berlin Heidelberg, New York, 1977).

[4] M.A. Krasnosel'skii, Positive Solutions of Operator Equations (Noordhoff, Grongingen, 1964). 